Article

\title{
Catalytic Activity of Oxidized Carbon Black and Graphene Oxide for the Crosslinking of Epoxy Resins
}

\author{
Maria Rosaria Acocella ${ }^{1, *}$, Carola Esposito Corcione ${ }^{2, *}$, Antonella Giuri ${ }^{2}$, Mario Maggio ${ }^{1}$, \\ Gaetano Guerra ${ }^{1}$ and Alfonso Maffezzoli ${ }^{2}$ \\ 1 Dipartimento di Chimica e Biologia e Unità di Ricerca INSTM, Università di Salerno, \\ 84084 Fisciano (SA), Italy; mariomaggiochem@gmail.com (M.M.); gguerra@unisa.it (G.G.) \\ 2 Dipartimento di Ingegneria dell'Innovazione, Università del Salento, 73100 Lecce, Italy; \\ antonella.giuri@unisalento.it (A.G.); alfonso.maffezzoli@unisalento.it (A.M.) \\ * Correspondence: macocella@unisa.it (M.R.A.); carola.corcione@unisalento.it (C.E.C.); \\ Tel.: +39-0899-69392 (M.R.A.); +39-0832-297326 (C.E.C.)
}

Academic Editor: Walter Remo Caseri

Received: 28 February 2017; Accepted: 3 April 2017; Published: 7 April 2017

\begin{abstract}
This article compares the catalytic activities of oxidized carbon black $(\mathrm{oCB})$ and graphene oxide (eGO) samples on the kinetics of a reaction of diglycidyl ether of bisphenol A (DGEBA) with a diamine, leading to crosslinked insoluble networks. The study is mainly conducted by rheometry and Differential Scanning Calorimetry (DSC). Following the same oxidation procedure, CB samples are more efficiently oxidized than graphite samples. For instance, $\mathrm{CB}$ and graphite samples with high specific surface areas (151 and $308 \mathrm{~m}^{2} / \mathrm{g}$ ), as oxidized by the Hummers' method, exhibit O/C wt/wt ratios of 0.91 and 0.62 , respectively. Due to the higher oxidation levels, these oCB samples exhibit a higher catalytic activity toward the curing of epoxy resins than fully exfoliated graphene oxide.
\end{abstract}

Keywords: carbon black oxidation; Differential Scanning Calorimetry; gel time

\section{Introduction}

Many recent studies have shown the catalytic activity of graphene oxide (eGO) [1-15] and graphene $(G)[16-18]$ toward numerous organic reactions. For several organic reactions, like the Mukayiama-Michael [7] or Friedel-Crafts reaction [18], similar catalytic activities have been observed for GO and G. In particular, for the Mukayiama-Michael reaction, it has been clearly established by DFT calculations that the catalytic activity is mainly due to supramolecular $\pi$-stacking interactions operating between the catalyst and the substrates [7]. For most organic reactions, like the Mukayiama-Aldol reaction [8] or esterification reactions [19], the catalytic activities of GO are definitely higher than for $\mathrm{G}$ (often negligible). In these cases, of course, the GO catalytic activity has been attributed to its functional groups, mainly those which are acidic.

The GO catalysis of ring-opening reactions in the amine crosslinking of epoxy resins $[10,20,21]$ is included in this second class. In fact, high specific surface area graphite, as well as highly reduced graphene oxide, present negligible catalytic activity.

Very recently, direct kinetic studies, including a full chemical characterization and quantitative evaluation of the low molecular mass products for reactions of diglycidyl ether of bisphenol A (DGEBA) with primary and secondary monoamines and alcohols, have shown that GO and exfoliated graphite oxide (eGO) are able to catalyze primary amine-epoxy, secondary amine-epoxy, and hydroxyl-epoxy additions [20]. In the same paper, it has been shown that non-oxidized graphene surfaces are only able to catalyze the first two reactions and not hydroxyl-epoxy additions, which are also needed for the completion of the crosslinking reaction [20]. 
For many decades, it has also been known that oxidized carbon black (oCB) is able to affect the cure behavior of epoxy resin systems [22,23]. In a recent paper, the WAXD patterns of oCB samples were interpreted by a disordered spatial arrangement of graphene oxide layers, exhibiting short in-plane correlation lengths [24].

In the present paper, the catalytic activity of eGO and oCB samples, exhibiting very different oxygen contents, in the amine crosslinking of DGEBA epoxy resins, the most industrially relevant epoxy resin, is compared. The possible catalytic behavior has been mainly explored by indirect phenomenological approaches [25], based on differential scanning calorimetry (DSC) and the rheological evaluation of viscosity increases.

\section{Materials and Methods}

\subsection{Materials}

Diglycidyl ether of bisphenol A (DGEBA), supplied by Resolution Performance Products (Houston, TX, USA) with the commercial name Epikote 828, is characterized by an epoxy equivalent weight (EEW) of $190 \mathrm{~g} /$ eq., corresponding to an average molecular weight (MwEpikote828) of $380 \mathrm{~g} / \mathrm{mol}$. Since neat DGEBA is characterized by an EEW $=170 \mathrm{~g} /$ eq., which corresponds to an average molecular weight (MwDGEBA) of $340 \mathrm{~g} / \mathrm{mol}$, as shown in Scheme 1, Epikote 828 is a mixture of neat DGEBA and its oligomers.

The curing agent, purchased by Sigma Aldrich (Milan, Italy), is isophorondiamine (IPDA), used without any further purification. IPDA is characterized by a Hydrogen Equivalent Weight, HEW $=43$. It was selected since it is a typical hardener used for the fabrication of epoxy-based composites. According to the stoichiometric ratio, 22 parts of hardener (i.e., 22 phr) were added to 100 parts of DGEBA. The molecular structure of the components of the thermoset resin, as well as the crosslinking conditions, are shown in Scheme 1.

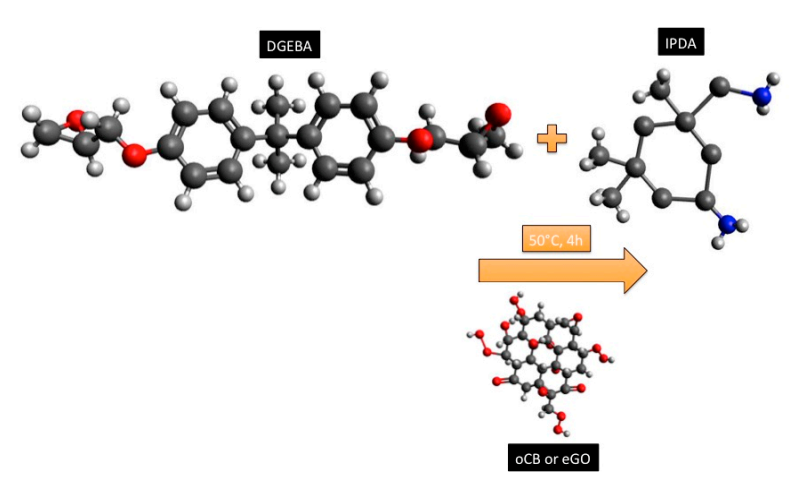

Scheme 1. Macromolecular structure of the reactants.

Synthetic Graphite $8427^{\circledR}$ with a high specific surface area (HSAG, of about $308 \mathrm{~m}^{2} / \mathrm{g}$ ) and a high shape anisotropy of the crystallites [26], was purchased from Asbury Graphite Mills Inc. (Asbury, NJ, USA).

The used carbon black samples, with specific surface areas of 36, 125, and $151 \mathrm{~m}^{2} / \mathrm{g}$, respectively, were purchased from Cabot (Boston, MA, USA).

\subsection{Preparation Procedures}

\subsubsection{Preparation of Oxidized Graphitic Materials}

Graphite Oxide (GO) and oxidized Carbon Black (oCB), were prepared by the Hummers' method of high specific surface area graphite and commercial carbon black samples [27]. A total of $120 \mathrm{~mL}$ of sulfuric acid and $2.5 \mathrm{~g}$ of sodium nitrate were introduced into a $2000 \mathrm{~mL}$ three-neck round 
bottomed flask immersed into an ice bath and $5 \mathrm{~g}$ of carbon was added, with magnetic stirring. After obtaining a uniform dispersion, $15 \mathrm{~g}$ of potassium permanganate was added to the sample very slowly, to minimize the risk of explosion. The reaction mixture was thus heated to $35^{\circ} \mathrm{C}$ and stirred for $24 \mathrm{~h}$. Deionized water $(700 \mathrm{~mL})$ was added to the resulting black and dark green slurry in small amounts, for carbon black and graphite, respectively, under stirring and, finally, $5 \mathrm{~mL}$ of $\mathrm{H}_{2} \mathrm{O}_{2}$ (30 wt \%) was gradually added. The obtained sample was poured into $7 \mathrm{~L}$ of deionized water, and then centrifuged at 10,000 rpm for 15 min with a Awel MF-48R centrifuge (Nuaire Plymouth, MN, USA). The isolated GO and oCB powders were washed twice with $100 \mathrm{~mL}$ of a $5 \mathrm{wt} \% \mathrm{HCl}$ aqueous solution, and subsequently washed with $500 \mathrm{~mL}$ of deionized water. Finally, powders were dried at $60{ }^{\circ} \mathrm{C}$ for $12 \mathrm{~h}$. About $6 \mathrm{~g}$ of all oCB samples and $7.5 \mathrm{~g}$ of GO powder were obtained. The obtained Oxygen/Carbon weight ratio was 0.62 for graphite oxide, and $0.16,0.83$, and 0.91 for oCB as obtained from CB samples exhibiting largely different BET specific surface areas (in the range 33-151 $\mathrm{m}^{2} / \mathrm{g}$ ) [24].

Exfoliated graphite oxide (eGO) samples were prepared in a planetary ball mill (Fritsch Pulverisette 7, (FRITSCH GmbH, Idar-Oberstein, Germany) for $2 \mathrm{~h}$, with a milling speed of $500 \mathrm{rpm}$, using $125 \mathrm{~mL}$ of GO powder which was added to a ceramic jar (inner diameter of $75 \mathrm{~mm}$ ) containing stainless steel balls (10 $\mathrm{mm}$ in diameter), with a ball-to-powder mass ratio of 10:1.

\subsubsection{Dispersion of Graphite-Based Nanofillers and the Cure of the Epoxy Resin}

The dispersion of the graphite-based fillers in the epoxy matrix was performed by adopting the solvent swelling method, previously reported in [28]. Each filler was dispersed in the polymer, according to the following steps. After the dispersion of each filler in acetone, the filler-solvent mixture was added to the epoxy resin and stirred for $5 \mathrm{~h}$ at $80^{\circ} \mathrm{C}$ and $400 \mathrm{rpm}$, until the complete evaporation of the solvent. The filler-epoxy mixture was subsequently degassed under vacuum at $T=60^{\circ} \mathrm{C}$. A total of $22 \mathrm{phr}$ of IPDA were finally added to the filler/resin mixture. A filler amount equal to $3 \mathrm{wt} \%$ was always added to the epoxy matrix.

\subsection{Characterization Techniques}

\subsubsection{Elemental Analysis}

Elemental analysis was performed with a Thermo FlashEA 1112 Series CHNS-O analyzer (Thermo Ficher Scientific, Waltham, MA, USA), after pretreating the samples in an oven at $100{ }^{\circ} \mathrm{C}$ for $12 \mathrm{~h}$.

\subsubsection{Wide-Angle X-ray Diffraction}

Wide-angle X-ray diffraction (WAXD) patterns were obtained by an automatic Bruker D8 Advance diffractometer (Bruker, MA, USA), in reflection, at $35 \mathrm{KV}$ and $40 \mathrm{~mA}$, using nickel filtered Cu-k $\alpha$ radiation (1.5418 $\AA$ ). The $d$-spacings were calculated using Bragg's law and the observed integral breadths ( $\beta$ obs) were determined by a fit with a Lorentzian function of the intensity corrected diffraction patterns. The instrumental broadening ( $\beta$ inst) was also determined by the fitting of the Lorentzian function to line profiles of a standard silicon powder 325 mesh (99\%). For each observed reflection, the corrected integral breadths were determined by subtracting the instrumental broadening of the closest silicon reflection from the observed integral breadths, $\beta=\beta$ obs $-\beta$ inst. The correlation lengths $(D)$ were determined using Scherrer's equation.

$$
D=\frac{K \lambda}{\beta \cos \theta}
$$

where $\lambda$ is the wavelength of the incident $X$-rays and $\theta$ is the diffraction angle, assuming the Scherrer constant $K=1$. 


\subsubsection{Thermogravimetric Analysis}

The thermogravimetric (TG) analysis was carried out on a TG $209 \mathrm{~F} 1$, manufactured by TA Instruments (Waters, MA, USA), from 20 to $800{ }^{\circ} \mathrm{C}$ at a heating rate of $10^{\circ} \mathrm{C} / \mathrm{min}$, under $20 \mathrm{~mL} / \mathrm{min}$ $\mathrm{N}_{2}$ flow.

\subsubsection{Infrared Spectroscopy}

The FTIR spectra were obtained at a resolution of $2.0 \mathrm{~cm}^{-1}$ with a FTIR -BRUKER Vertex70, (Bruker, MA, USA) spectrometer equipped with a deuterated triglycine sulfate (DTGS) detector and a $\mathrm{KBr}$ beam splitter, using $\mathrm{KBr}$ pellets prepared by mixing $1 \mathrm{mg}$ of sample in $100 \mathrm{mg}$ of $\mathrm{KBr}$. The frequency scale was internally calibrated to $0.01 \mathrm{~cm}^{-1}$ using a He-Ne laser. A total of 32 scans were signal averaged to reduce the noise. The spectra acquisition was in the $4000-450 \mathrm{~cm}^{-1}$ wavenumber range.

\subsubsection{BET Specific Surface Areas}

Specific surface areas of the carbon and oxidized carbon samples were measured by nitrogen adsorption at liquid nitrogen temperature $(77 \mathrm{~K})$ with a Nova Quantachrome 4200e instrument. Before the adsorption measurements, samples were degassed at $60^{\circ} \mathrm{C}$ under vacuum for $24 \mathrm{~h}$. The specific surface area values were determined by using 11-point BET analysis (Quantachrome Instruments, Boynton Beach, FL, USA).

\subsubsection{Rheometry}

The rheological properties of the filled and unfilled mixtures were evaluated by monitoring the evolution of the storage modulus, $\mathbf{G}^{\prime}$, and the loss modulus, $\mathbf{G}^{\prime \prime}$, as functions of time. Measurements were made using a strain-controlled rheometer (Ares, Rheometric Scientific, Piscataway, NJ, USA), adopting a parallel plate geometry characterized by plates with a radius of $12.5 \mathrm{~mm}$. Three isothermal tests at $50{ }^{\circ} \mathrm{C}$ were performed on each sample, using a frequency of $1 \mathrm{~Hz}$ and a deformation of $10 \%$.

The dynamic-mechanical properties of a curing system depend on the degree of reaction, dramatically changing when the gel point is approached. The time to gelation or gel time $\left(t_{\mathrm{g}}\right)$ was determined according to the literature, as the cross between the $\mathbf{G}^{\prime}$ and $\mathbf{G}^{\prime \prime}$ curves [29,30]. Each test was performed until gelation was observed.

Finally, it must be underlined that the gel time is observed for a constant value of the degree of the reaction, according to Flory [31], and hence it represents a fast way to compare the rate of the reaction of thermosetting resins with the same reagents and under different conditions, such as those considering temperature or catalysis.

\subsubsection{Differential Scanning Calorimetry}

The curing reaction of filled and unfilled epoxy resin was measured using a differential scanning calorimeter (DSC) supplied by Mettler Toledo 622 (Columbus, OH, USA). Dynamic DSC scans were performed on liquid samples from 20 to $250{ }^{\circ} \mathrm{C}$ at $10^{\circ} \mathrm{C} / \mathrm{min}$, under nitrogen atmosphere. Isothermal DSC scans were also performed at $50{ }^{\circ} \mathrm{C}$ for $4 \mathrm{~h}$, under nitrogen atmosphere, performing at least three repetitions. The reaction was considered to be complete when the heat flow curve leveled off to a constant value. The area under the exothermal curve, based on an extrapolated horizontal baseline aligned to the asymptotic value of the DSC signal at the end of the reaction, was used to calculate the heat of the reaction, $\Delta H(\mathrm{~J} / \mathrm{g})$, at the test temperature.

\section{Results}

\subsection{Characterization of the Carbon Nanofillers}

An elemental analysis of the carbon-based nanofillers used in the present study is reported in Table 1. 
Table 1. Elemental analysis of the carbon-based nanofillers used in the study ${ }^{a}$.

\begin{tabular}{|c|c|c|c|c|c|}
\hline Nanofiller & $\begin{array}{c}C \\
(w t \%)\end{array}$ & $\begin{array}{c}\mathrm{H} \\
\text { (wt \%) }\end{array}$ & $\begin{array}{c}\mathrm{O} \\
(w t \%)\end{array}$ & $\begin{array}{c}S \\
(w t \%)\end{array}$ & $\mathrm{O} / \mathrm{C}$ \\
\hline oCB-1 & 85.9 & 0.2 & 13.75 & $<0.2$ & 0.16 \\
\hline oCB-2 & 50.3 & 2.3 & 41.7 & 5.4 & 0.83 \\
\hline oCB-3 & 40.8 & 1.73 & 37.42 & 9.9 & 0.91 \\
\hline eGO & 59.4 & 0.6 & 37.1 & 2.6 & 0.62 \\
\hline
\end{tabular}

a Elemental composition of anhydrous samples: The error in $\mathrm{C}$ and $\mathrm{O}$ content is close to $1 \mathrm{wt} \%$, while the error in $\mathrm{H}$ content is close to $0.1 \mathrm{wt} \%$; water content (as evaluated by TGA, after equilibration at room temperature in air with a relative humidity of $45 \%$ ) was in the range of $\pm 2 \mathrm{wt} \%$, for all the examined samples.

In agreement with a previous report [24], the $\mathrm{O} / \mathrm{C}$ ratio of the considered oCB samples, for the same oxidation procedure, markedly increases $(0.16,0.83$, and 0.91$)$ with the specific surface area of the starting CB samples (36, 125, and $151 \mathrm{~m}^{2} / \mathrm{g}$, respectively), with an average size of 50-100 $\mathrm{nm}$. These three oCB samples thereafter will be named oCB-1, oCB-2, and oCB-3, respectively. For the same oxidation procedure, the eGO sample, i.e., the graphene oxide sample as obtained by the ball-milling of graphite oxide, exhibits a rather low $\mathrm{O} / \mathrm{C}$ ratio $(0.62)$, irrespective of the very high specific surface area of the starting graphite $\left(308 \mathrm{~m}^{2} / \mathrm{g}\right)$.

The large difference in the degree of oxidation of the considered nanofillers is also clearly shown by the TGA scans of Figure 1.

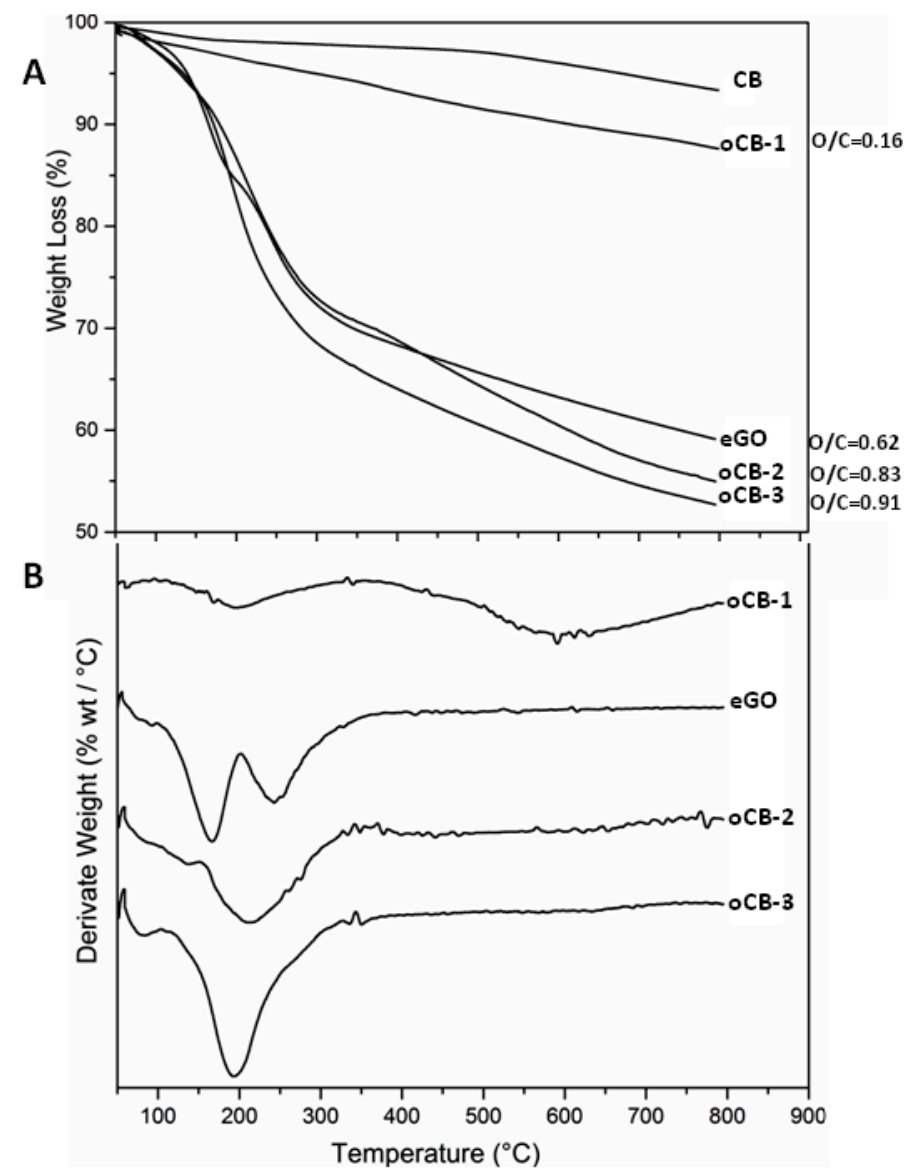

Figure 1. TGA scans at $10{ }^{\circ} \mathrm{C} / \mathrm{min}$ after drying at $100{ }^{\circ} \mathrm{C}$ in the TGA apparatus, of starting Carbon black (CB) and all the considered carbon nanofillers: oCB-1; eGO; oCB-2; oCB-3. (A) TGA; (B) DTGA. Close to each curve, their $\mathrm{O} / \mathrm{C}$ molar ratio is indicated. 
Information relative to the functional groups present in the different nanofillers is obtained from the FTIR spectra (Figure 2).

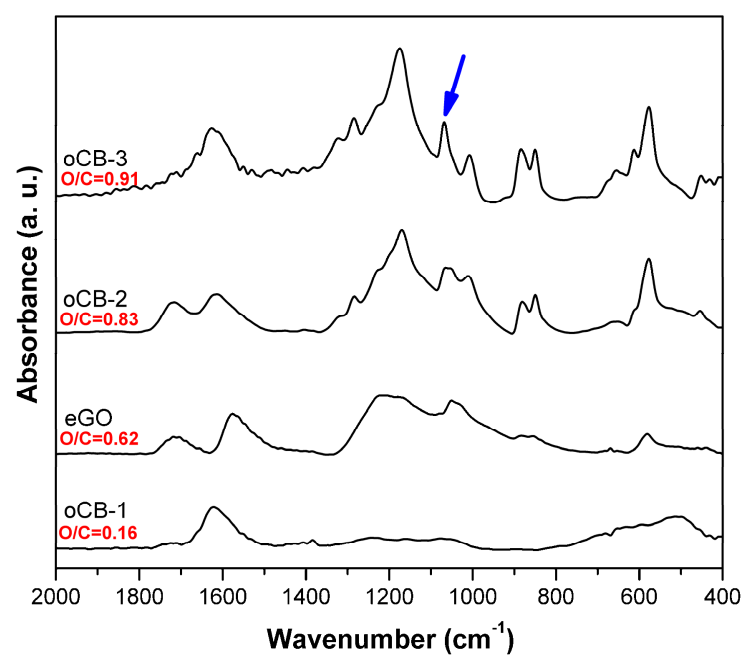

Figure 2. FTIR spectra in the range $2000-400 \mathrm{~cm}^{-1}$ of the considered carbon nanofillers: oCB-1; eGO; oCB-2; oCB-3. Close to each curve, their O/C molar ratio is indicated.

In particular, as the $\mathrm{O} / \mathrm{C}$ ratio increases, many vibrational peaks appear in the $1300-900 \mathrm{~cm}^{-1}$ range (mainly referring to the $\mathrm{C}-\mathrm{O}$ stretching of the ether, epoxy, alcoholic, and carboxylic groups), becoming progressively more defined. Moreover, the well-defined vibrational peak located at $1069 \mathrm{~cm}^{-1}$ (indicated with an arrow in Figure 2) reveals the contribution of an increased amount of the sulfonic group [32,33].

Information on the structural organization of the oxidized samples has also been obtained by WAXD. Figure 3 compares the WAXD patterns of the graphene oxide sample and of the carbon black sample with the highest specific surface area and of the corresponding mostly oxidized sample (oCB-3). The WAXD pattern of graphene oxide (lower pattern in Figure 3) shows the maintenance of hk0 (110 and 100) graphite reflections and the presence of a broad halo centred at $2 \theta=24^{\circ}$. This indicates the presence of an in-plane graphitic order and the nearly complete absence of a packing order between the graphene oxide layers.
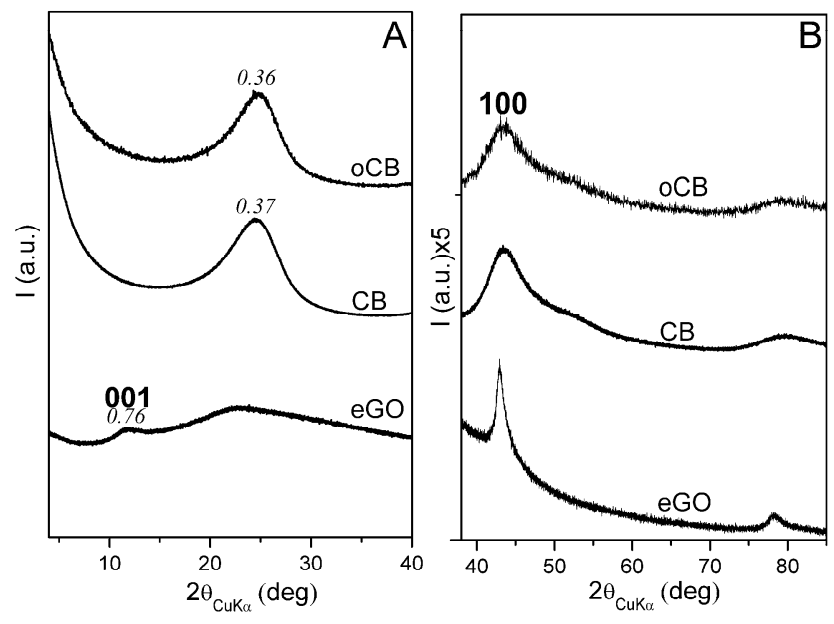

Figure 3. X-ray diffraction patterns $(\mathrm{CuK} \alpha)$ of: graphene oxide (eGO, with $\mathrm{O} / \mathrm{C}$, wt $/ \mathrm{wt}=0.62)$; $\mathrm{CB}$ sample with the highest BET specific surface area; this $\mathrm{CB}$ after oxidation $(\mathrm{oCB}-3, \mathrm{O} / \mathrm{C}$, wt $/ w t=0.91)$. Patterns in the $2 \theta$ range $4-40^{\circ}(\mathbf{A})$ and enlarged patterns in the $2 \theta$ range $40-85^{\circ}$ (B). 
The WAXD pattern of the CB sample with the highest BET specific surface area (intermediate pattern in Figure 3) is very similar to those of all the considered carbon black samples and poorly changes after extensive oxidation up to $\mathrm{O} / \mathrm{C}=0.91$ (upper pattern in Figure 3) [24]. As expected, the in-plane graphitic order for the $\mathrm{oCB}$ samples is much lower than for the eGO sample. For instance, the in-plane correlation length (as evaluated from the broadness of the 100 reflection) decreases from $11.1-11.3 \mathrm{~nm}$ to $2.1-2.3 \mathrm{~nm}$, passing from GO to oCB. The amorphous halo, on the contrary, is definitely broader for the GO sample rather than for the oCB samples, with the half-height width reducing from $10.1^{\circ}$ to $4.5^{\circ}$. The occurrence of amorphous halos which are much broader for eGO than for $\mathrm{CB}$ and $\mathrm{OCB}$, could be rationalized by a broader distribution of interatomic distances, which is possibly determined by the disordered spatial disposition of the larger layers.

\subsection{Gel Time of the Epoxy Resin Crosslinking Reaction by the Rheometer}

Carbon nanofillers thoroughly characterized in the previous section have been tested in model reactive epoxy resins, close to those used in practical applications, as possible catalysts for the crosslinking of DGEBA epoxy resins with amines. The cure reaction was studied by isothermal rheological measurements, at $50^{\circ} \mathrm{C}$. The storage modulus, $\mathrm{G}^{\prime}$ (empty symbols), and the loss modulus, $\mathrm{G}^{\prime \prime}$ (full symbols), of unfilled and filled compounds, as determined by forced harmonic oscillation measurements, are reported in Figure 4 as functions of time.

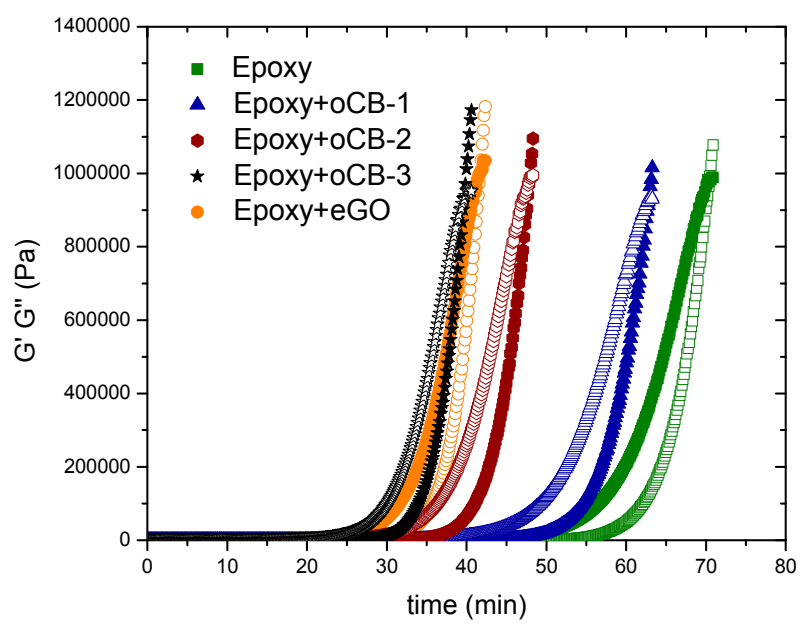

Figure 4. Storage modulus ( $\mathrm{G}^{\prime}$, empty symbols) and loss modulus $\left(\mathrm{G}^{\prime \prime}\right.$, full symbols) versus time for the DGEBA-IPDA epoxy resin, pure (green) or with $3 \mathrm{wt} \%$ of different carbon nanofillers: oCB-1 (O/C, $\mathrm{wt} / \mathrm{wt}=0.16)($ blue $) ; \mathrm{oCB}-2(\mathrm{O} / \mathrm{C}, \mathrm{wt} / \mathrm{wt}=0.83$ (wine); oCB-3 $(\mathrm{O} / \mathrm{C}, \mathrm{wt} / \mathrm{wt}=0.91)($ black$) ; \mathrm{eGO}(\mathrm{O} / \mathrm{C}$, $w t / w t=0.62)$ (orange).

The gel time $\left(t_{\mathrm{gel}}\right)$, measured as the cross between the $\mathbf{G}^{\prime}$ and $\mathbf{G}^{\prime \prime}$ curves of the neat resin and of the nanocomposites, is reported in Table 2.

Table 2. Gel time $\left(t_{\text {gel }}\right)$, measured at the intersection of the $\mathbf{G}^{\prime}$ and $\mathbf{G}^{\prime \prime}$ curves of the neat resin and of the nanocomposites.

\begin{tabular}{cc}
\hline Sample & $\boldsymbol{t}_{\text {gel }}[\mathrm{min}]$ \\
\hline Epoxy & $70.5 \pm 5.6$ \\
Epoxy oCB-1 & $62.9 \pm 4.4$ \\
Epoxy oCB-2 & $47.9 \pm 3.6$ \\
Epoxy oCB-3 & $39.7 \pm 2.0$ \\
Epoxy_eGO & $41.7 \pm 2.1$ \\
\hline
\end{tabular}


The gel time of the neat epoxy resin $\left(t_{\mathrm{g}}=70.5 \mathrm{~min}\right)$ is progressively reduced with the degree of oxidation of $\mathrm{oCB}$ and is nearly halved in the presence of the $\mathrm{oCB}$ sample with $\mathrm{O} / \mathrm{C}=0.91\left(t_{\mathrm{g}}=39.7 \mathrm{~min}\right)$. An effective reduction of the gel time is also observed with eGO $\left(\mathrm{O} / \mathrm{C}=0.62, t_{\mathrm{g}}=41.7 \mathrm{~min}\right)$. The gel time represents the time to reach a given value of the degree of reaction once the stoichiometry is defined. In this case, according to the Flory equation [31], the reaction of a bifunctional epoxy monomer with a tetrafunctional amine (neglecting side reactions with $\mathrm{OH}$ groups) leads to a degree of reaction at gelation of 0.58 . Since the lower the gel time, the higher the reaction rate, these data clearly confirm a catalytic activity of the oxidized groups on the graphitic layers on the epoxy crosslinking.

\subsection{DSC Heating Scans of Unfilled and Filled Epoxy Resins}

DSC scans, performed on the neat epoxy resin and on resins filled with $3 \mathrm{wt} \%$ of the considered carbon nanofillers, at a heating rate of $10^{\circ} \mathrm{C} / \mathrm{min}$, are compared in Figure 5.

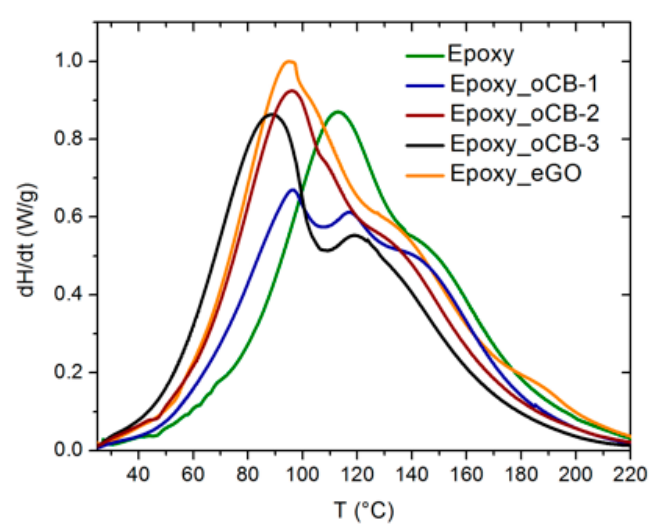

Figure 5. DSC scans (heating rate $=10^{\circ} \mathrm{C} / \mathrm{min}$ ) for the pure epoxy resin (green) and for composite resins with 3 wt \% of: oCB-1 (blue); oCB-2 (wine); oCB-3 (black); eGO (orange).

The scan of the neat resin (green curve in Figure 5) presents, beside the principal exothermic peak at $114{ }^{\circ} \mathrm{C}$, a shoulder (roughly located at $145^{\circ} \mathrm{C}$ ) that could be possibly due to a hydroxyl-epoxy addition (etherification) reaction [20]. For the compound with the poorly oxidized CB sample (oCB-1), two peaks are clearly distinguished, with a more intense one at $96{ }^{\circ} \mathrm{C}$, which clearly indicates the occurrence of some catalysis toward the etherification reaction $[10,20]$. In the absence of any catalyst, the amine addition is strongly favored with respect to etherification. However, oxidized graphites are catalysts of both reactions, as formerly demonstrated $[10,20]$, acting first on primary and secondary amine additions, and then on etherification. This last reaction occurs when a gelled network is formed and hence it is also affected by the mobility of chain segments, which depends on the degree of crosslinking. Therefore, when more amine hydrogens react (first peak in Figure 5), more hydroxyl are produced, but also more steric hindrance is expected at higher temperatures, when etherification is favored. Therefore, the intensity of the first peak progressively increases at the expense of the higher temperature peaks as the oxidation degree of the carbon nanofillers increases. Moreover, for the mostly oxidized sample (oCB-3), this peak corresponding to the amine catalyzed reaction is also centered at the lowest temperature $\left(\approx 89^{\circ} \mathrm{C}\right)$. The fraction of the heat of the reaction associated with the amine catalyzed reaction, assessed by peak deconvolution for the DSC scans of Figure 5, is shown in Table 3. The observed shift to lower temperatures of the first exothermic peak indicates a relevant catalytic activity of graphite oxide toward the amine-epoxy reaction.

For the exothermic phenomena of Figure 5, the total heat of the crosslinking reaction per mass of resin $\left(\Delta H_{\mathrm{U}}\right)$ and the position of the main peak $\left(T_{\text {peak }}\right)$ are also listed in Table 3.

It is apparent that the $T_{\text {peak, }}$, as well as the heat of the reaction, increases with the degree of oxidation of the carbon nanofillers. The number of reacte epoxy groups, indicated by $\Delta H_{\mathrm{U}}$ in Table 3 , also increases with the degree of oxidation of nanographite. 
Table 3. Results of DSC scans at $10{ }^{\circ} \mathrm{C} / \mathrm{min}$, for the neat epoxy resin and epoxy resins with $3 \mathrm{wt} \%$ of different carbon nanofillers: enthalpy changes, normalized with respect to the resin weight $\left(\Delta H_{\mathrm{U}}\right)$, peak temperature $\left(T_{\text {peak }}\right)$, and fraction of catalyzed reaction.

\begin{tabular}{cccc}
\hline Sample & $\boldsymbol{\Delta} \boldsymbol{H}_{\mathbf{U}}(\mathbf{J} / \mathbf{g})$ & $\boldsymbol{T}_{\text {peak }}{ }^{\circ} \mathbf{C}$ & Amine Catalyzed Fraction (\%) \\
\hline Epoxy & $313.9 \pm 1.1$ & $113.7 \pm 0.9$ & 0 \\
Epoxy + oCB-1 $(\mathrm{O} / \mathrm{C}=0.16)$ & $327.5 \pm 2.2$ & $96.5 \pm 0.8$ & 12 \\
Epoxy + oCB-2 $(\mathrm{O} / \mathrm{C}=0.83)$ & $383.8 \pm 1.1$ & $96.2 \pm 0.7$ & 34 \\
Epoxy + oCB-3 $(\mathrm{O} / \mathrm{C}=0.91)$ & $352.5 \pm 1.7$ & $88.7 \pm 1.1$ & 56 \\
Epoxy $+\mathrm{eGO}(\mathrm{O} / \mathrm{C}=0.62)$ & $374.4 \pm 2.3$ & $95.0 \pm 0.7$ & 29 \\
\hline
\end{tabular}

\subsection{Kinetics of the Epoxy Resin Crosslinking Reaction by Isothermal DSC}

Isothermal DSC scans at $50{ }^{\circ} \mathrm{C}$, for the considered epoxy resin, without fillers and with $3 \mathrm{wt} \%$ of different graphite-based nanofillers, are reported in Figure 6. A short time interval, usually about $30 \mathrm{~s}$, is needed for temperature stabilization after the ramp at $40{ }^{\circ} \mathrm{C} / \mathrm{min}$ used to reach the isothermal test temperature. The transition between dynamic and isothermal conditions is responsible for a jump in the heat flow, which means that the data obtained in the first $30 \mathrm{~s}$ of the DSC test are not reliable. For this reason, all isothermal curves were corrected, neglecting the data point in the first $30 \mathrm{~s}$ after the curing temperature is reached, and determining by linear extrapolation of the data points recorded between 30 and $60 \mathrm{~s}$, the value of the heat flow at time $=0$. These corrected isothermal DSC scans are shown in Figure 6 and the isothermal heat of the reaction and the time to reach the peak, calculated according to the procedure described in the Experimental section, are listed in Table 4, together with the residual heat of the reaction $\left(\Delta H_{\text {residual }}\right)$ and the glass transition temperature $\left(T_{\mathrm{g}}\right)$ measured from a dynamic scan performed after the isothermal scan.

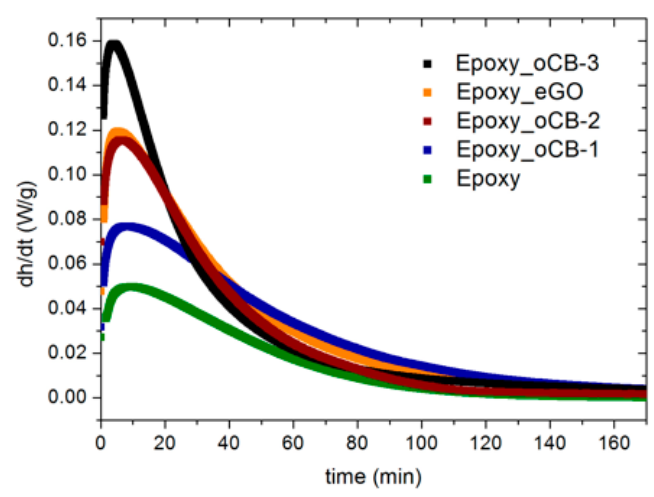

Figure 6. Isothermal DSC scans at $50^{\circ} \mathrm{C}$ of the DGEBA-IPDA epoxy resin. The lowest curve (green) corresponds to the neat epoxy resin. The other curves correspond to the epoxy resin filled by $3 \mathrm{wt} \%$ of different nanofillers: oCB-1 $(\mathrm{O} / \mathrm{C}$, wt $/ \mathrm{wt}=0.16)($ blue $)$; oCB-2 $(\mathrm{O} / \mathrm{C}$, wt $/ w t=0.83)($ wine $)$ oCB-3 $(\mathrm{O} / \mathrm{C}, \mathrm{wt} / \mathrm{wt}=0.91)($ black$) ; \mathrm{eGO}(\mathrm{O} / \mathrm{C}, \mathrm{wt} / \mathrm{wt}=0.62)$ (orange).

Table 4. Enthalpy, normalized with respect to the resin weight $(\Delta H)$ and peak time $\left(t_{\text {peak }}\right)$ obtained by isothermal DSC scans at $50{ }^{\circ} \mathrm{C}$, for the neat epoxy resin and the epoxy resin with different graphite-based nanofillers.

\begin{tabular}{ccccc}
\hline Sample & $\boldsymbol{\Delta} \boldsymbol{H}(\mathbf{J} / \mathbf{g})$ & $\boldsymbol{t}_{\text {peak }}(\mathbf{s})$ & $\boldsymbol{\Delta} \boldsymbol{H}_{\text {residual }}(\mathrm{J} / \mathbf{g})$ & $\boldsymbol{T}_{\mathbf{g}}\left({ }^{\circ} \mathbf{C}\right)$ \\
\hline Epoxy & $158.0 \pm 2.0$ & $508 \pm 1$ & $74.6 \pm 1.1$ & 54.7 \\
Epoxy + oCB-1 & $306.0 \pm 18.0$ & $504 \pm 19$ & $18.1 \pm 0.9$ & 66.3 \\
Epoxy + oCB-2 & $297.7 \pm 5.7$ & $393 \pm 6$ & $62.6 \pm 1.3$ & 59.4 \\
Epoxy + oCB-3 & $337.0 \pm 27.0$ & $249 \pm 63$ & $13.4 \pm 0.4$ & 64.6 \\
Epoxy + eGO & $337.0 \pm 2.0$ & $306 \pm 1$ & $26.2 \pm 0.8$ & 63.2 \\
\hline
\end{tabular}


As already observed for the gel time data of Table 3, the peak time $\left(t_{\text {peak }}\right)$ and the residual heat of the reaction of Table 4 are significantly reduced in the presence of eGO, oCB-1, and oCB-3. This further indicates the catalytic activity of oxidized graphitic layers on epoxide-amine reactions.

Compounds with graphite-based nanofillers are also characterized by a significantly higher heat of reaction: neat epoxy resin is characterized by a reaction enthalpy of $158 \mathrm{~J} / \mathrm{g}$, increasing to $306 \mathrm{~J} / \mathrm{g}$ when oCB- 1 is added and $337 \mathrm{~J} / \mathrm{g}$ in the presence of eGO and oCB-3. An increase of about $93 \%$ is already observed with oCB-1, i.e., the less oxidized CB. Because the overall crosslinking enthalpy for the unfilled and filled epoxy resins, as determined by DSC heating scans, are all in the range $310-380 \mathrm{~J} / \mathrm{g}$ (see, next section), the low reaction enthalpy of the neat epoxy resin clearly indicates that in the absence of the carbon-based fillers, less than $50 \%$ of the crosslinking reaction occurs, even after long term treatments at $50{ }^{\circ} \mathrm{C}$. Isothermal curing at $50{ }^{\circ} \mathrm{C}$ leads to vitrification, which is responsible for the end of the reaction, which depends on free volume relaxation [34-38], delayed with respect to chemical conversion when faster reactions occur, i.e., when graphite oxide is present. The graphite-based fillers promote an increase of the $T_{\mathrm{g}}$ of the neat resin from 54.7 to $64.6^{\circ} \mathrm{C}$ for Epoxy $+\mathrm{oCB}-3$; this last behavior influences the kinetics reaction, as confirmed by the decreasing of the residual heat of the reaction from 74.6 (neat resin) to $13.4 \mathrm{~J} / \mathrm{g}$ (Epoxy + oCB-3). It is important to note that the sum of $\Delta H$ (measured

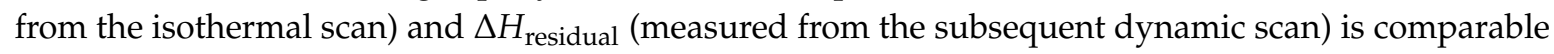
to the value of $\Delta H_{\mathrm{U}}$ (measured from the dynamic scan) reported in Table 3.

In order to obtain further insight into the reaction mechanism, the rate of reaction, $d \alpha / d t$, was calculated from the DSC scans, as:

$$
\frac{d \alpha}{d t}=\frac{1}{H_{U}}\left(\frac{d H}{d t}-B L\right)
$$

where $B L$ is the baseline, set as described above; $d H / d t$ is the heat flow for unit mass shown in Figure 7; $\Delta H_{\mathrm{U}}$ is the total heat of the reaction obtained by the integration of each peak of the dynamic scan reported in Figure 5, adopting the same baseline [39]; $\alpha$ represents the actual degree of reaction; and $d \alpha / d t$ is the rate of the reaction. $\alpha$ represents a phenomenological measure of the advancement of the curing reaction and is obtained by the integration of Equation (1). A comparison between the $d \alpha / d t$ experimental curves obtained by applying Equation (1) to the DSC scans of each system, is reported in Figure 7.

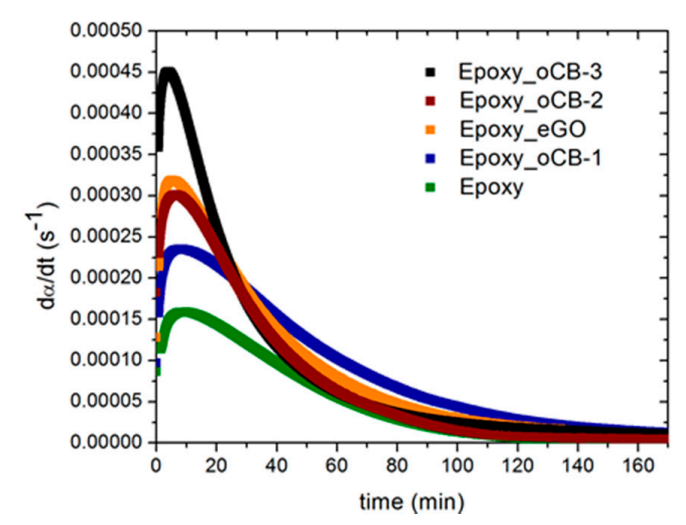

Figure 7. Comparison between $d \alpha / d \mathrm{t}$ experimental curves of each system: epoxy resin filled by $3 \mathrm{wt} \%$ of different nanofillers: oCB-1 $(\mathrm{O} / \mathrm{C}, \mathrm{wt} / \mathrm{wt}=0.16)($ blue $)$; oCB-2 $(\mathrm{O} / \mathrm{C}, \mathrm{wt} / \mathrm{wt}=0.83)($ wine $)$; oCB-3 $(\mathrm{O} / \mathrm{C}, \mathrm{wt} / \mathrm{wt}=0.91)($ black$) ; \mathrm{eGO}(\mathrm{O} / \mathrm{C}, \mathrm{wt} / \mathrm{wt}=0.62)$ (orange).

Multiple events may occur simultaneously and lead to a very complicated reaction, and consequently, the use of multiple rate constants can provide more accurate modeling results. Kamal's model [40] involves two rate constants and has been successfully applied to model a variety of resins:

$$
\frac{d \alpha}{d t}=\left(k_{1}+k_{2} \alpha^{m}\right)(1-\alpha)^{n}
$$


where $m$ and $n$ are the fitting parameters which do not depend on temperature, and $k_{1}$ and $k_{2}$ are temperature-dependent rate constants. The validity of Equation (3) is limited to the reactions for which the kinetics of bond formation is the only rate-controlling step in the curing process. While this is usually true in the early stage, species diffusion can become very slow and govern the curing reaction rate near and above the glass transition. To take into account the different cure rate controlling mechanisms and achieve a greater accuracy at high conversions, some modifications of the available cure kinetics models have been introduced by Cole [41,42], by adding a term to explicitly account for the shift from kinetics to diffusion control in an autocatalytic thermosetting resin system. The modified expression has the following form:

$$
\frac{d \alpha}{d t}=\frac{k \alpha^{m}(1-\alpha)^{n}}{1+e^{c\left\{\alpha-\left(\alpha_{c 0}+\alpha_{c t} T\right)\right\}}}
$$

where $c$ is the diffusion constant and $\alpha_{c 0}$ is the critical degree of cure at a temperature of absolute zero. The constant $\alpha_{c t}$ accounts for an increase in the critical resin degree of cure with temperature. $K, m$, and $n$ follow the same definitions as in previous equations. Both Cole and Kenny et al. [40-42], proposed a simpler way to account for vitrification as the cause of the end of a reaction at low curing temperatures $[43,44]$ :

$$
\frac{d \alpha}{d t}=\left(k_{1}+k_{2} \alpha^{m}\right)\left(\alpha_{\max }-\alpha\right)^{n}
$$

where $\alpha_{\max }$ is the maximum degree of cure at a given temperature due to the vitrification phenomenon observed in isothermal cure. The constants $\mathrm{m}$ and $\mathrm{n}$ are the reaction orders which do not depend on temperature, to be experimentally determined, and $k_{1}$ and $k_{2}$ are temperature-dependent rate constants. Equation (4) incorporates the term $\alpha_{\max }$, so that the fractional conversion will not exceed the degree of cure associated with vitrification at a specific temperature. The isothermal rate of reaction data, reported in Figure 7, were interpolated with Equation (4), calculating $\alpha_{\max }$, from:

$$
\alpha_{\max }=\frac{\Delta H_{\max }}{\Delta H_{\mathrm{U}}}
$$

where $\Delta H_{\max }$ is the maximum heat of the reaction calculated from the isothermal scan, and $\Delta H_{\mathrm{U}}$ is the ultimate heat released during a complete reaction, measured from the dynamic scan.

The constant $k_{1}$ represents the initial rate of the reaction, i.e., $k_{1}=d \alpha / d t$ at $t=0 . k_{1}$, readily obtained from experimental DSC data, and is reported for each sample in Table 5. A comparison between the kinetic model predictions and the experimental data for unfilled and filled systems, reported in Figure 8 , shows a good agreement. The peak value, $(d \alpha / d t)_{\text {peak }}$, is reported for each experimental system in Table 5.

The parameter $m$ in Equation (5) is representative of the increase of the rate of the reaction at the beginning of the reaction (low values of $\alpha$ ), before the peak. On the other hand, the parameter $n$ in Equation (4) is dominant in the final part of the reaction (high values of $\alpha$ ) [45] The autocatalytic effect, as measured by the low values of $\mathrm{m}$, is higher than that of neat epoxy when oxidized graphites are used.

Table 5. $d \alpha / d t_{\text {peak }}\left(\mathrm{s}^{-1}\right)$ and kinetic model parameters.

\begin{tabular}{ccccccc}
\hline Sample & $(\boldsymbol{d} \boldsymbol{\alpha} / \boldsymbol{d} \boldsymbol{t})_{\mathbf{p e a k}}\left(\mathbf{s}^{\mathbf{- 1}}\right)$ & $\boldsymbol{\alpha}_{\mathbf{m a x}}$ & $\boldsymbol{m}$ & $\boldsymbol{n}$ & $\boldsymbol{k}_{\mathbf{1}}\left(\mathbf{s}^{\mathbf{- 1}}\right) \times \mathbf{1 0}^{\mathbf{6}}$ & $\boldsymbol{k}_{\mathbf{2}}\left(\mathbf{s}^{-\mathbf{1}}\right) \times \mathbf{1 0}^{\mathbf{6}}$ \\
\hline Epoxy & $1.57 \times 10^{-4}$ & 0.51 & 0.51 & 1.06 & 204 & 650 \\
Epoxy + oCB-1 & $2.33 \times 10^{-4}$ & 0.94 & 0.21 & 1.07 & 740 & 340 \\
Epoxy + OCB-2 & $3.02 \times 10^{-4}$ & 0.78 & 0.39 & 1.22 & 230 & 630 \\
Epoxy + oCB-3 & $4.49 \times 10^{-4}$ & 0.96 & 0.30 & 1.58 & 230 & 670 \\
Epoxy + eGO & $3.18 \times 10^{-4}$ & 0.90 & 0.20 & 1.22 & 990 & 490 \\
\hline
\end{tabular}


(a)

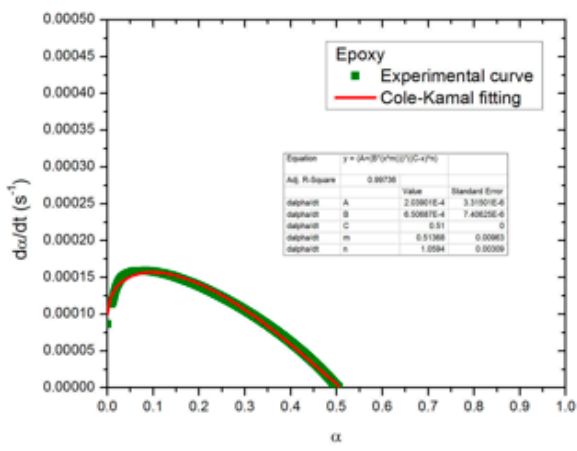

(b)

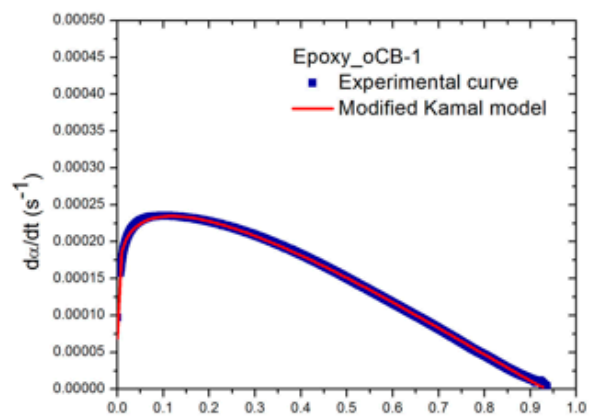

(d)

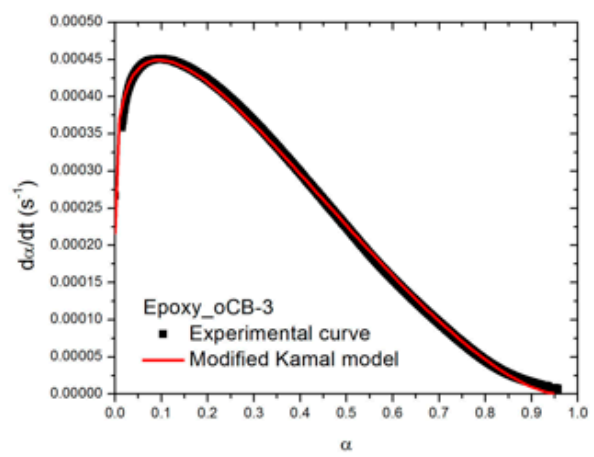

(c)

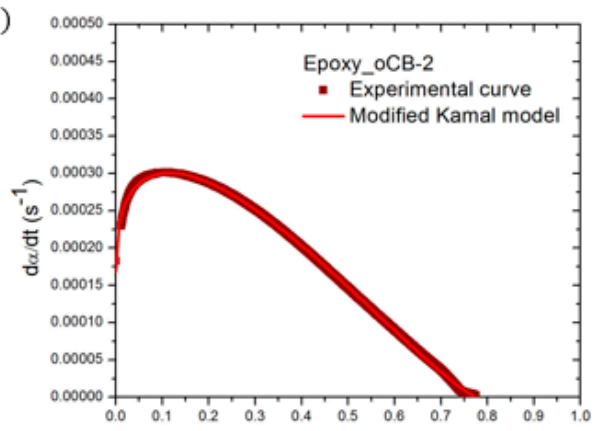

(e)

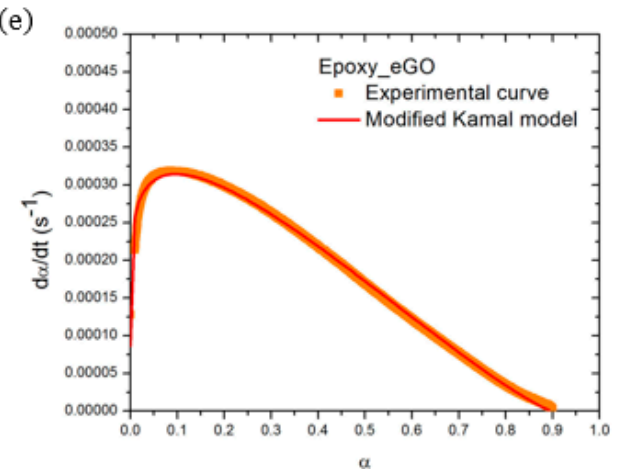

Figure 8. Comparison between Equation (6) model predictions and the experimental data of epoxy resins (a) filled by $3 \mathrm{wt} \%$ of different nanofillers; (b) oCB-1 (O/C, wt/wt $=0.16)$; (c) oCB-2 $(\mathrm{O} / \mathrm{C}$, $w t / w t=0.83) ;(d) o C B-3(O / C, w t / w t=0.91) ;(e) e G O(O / C, w t / w t=0.62)$.

The parameter $\mathrm{n}$ shown in Table 5 increases, changing from a value of about one to values higher than one when nanofillers are added to the resin, in particular, the highest value of $n$ was found when oCB-3 is used. A possible explanation for this kinetic change can be addressed by looking at the differences in the peak time and the heat of the reaction. When the rate of the reaction is very fast, vitrification, responsible for the end of the reaction at this temperature, is delayed as a consequence of non-equilibrium conditions, corresponding to a difference between the actual and the equilibrium free volume, as occurs in a crosslinked system undergoing fast reactions [34-38]. At a high enough rate of reaction, the actual specific volume is not capable of following the equilibrium specific volume, corresponding to the instantaneous molecular configuration which depends on the amount of reacted groups. This effect explains why, in a faster reaction, a higher heat of reaction, i.e., a larger amount of reacted groups, is observed as a consequence of a delay in vitrification (see also $\Delta H$ in Table 4 ). Furthermore, at a higher rate of reaction, the specific volume is farther from its equilibrium value, and, close to the end of the reaction, this leads to a more rapid decrease in the rate of the reaction as a consequence of the vitrification driven reaction end point. The rate of the reaction decreases faster 
when $n$ is higher: this is also evident from the last portion of the rate of reaction curves shown in Figure 7 (time $>30 \mathrm{~min}$ ).

\section{Conclusions}

The influence of different graphite-based nanofillers (exfoliated graphite oxide and oxidized carbon black) on the epoxide ring opening reactions of diglycidyl ether of bisphenol A (DGEBA), the most used component of commercial epoxy resins, as induced by amines, has been studied.

For the same oxidation procedure, $\mathrm{CB}$ samples are more efficiently oxidized than graphite samples. For instance, $\mathrm{CB}$ and graphite samples with a high specific surface area (151 and $308 \mathrm{~m}^{2} / \mathrm{g}$ ), as oxidized by the Hummers' method, exhibit $\mathrm{O} / \mathrm{C}$ wt/wt ratios equal to 0.91 and 0.62 , respectively.

Rheological data at $50{ }^{\circ} \mathrm{C}$ confirm the occurrence of a catalytic behavior. In fact, the gel time is reduced by $41 \%$ and $44 \%$ in the presence of $3 \mathrm{wt} \%$ graphene oxide (eGO) and highly oxidized CB (oCB-3), respectively.

Isothermal DSC studies at $50{ }^{\circ} \mathrm{C}$ show that, correspondingly, the time for the exothermic peak is reduced for eGO and oCB-3 by $40 \%$ and $51 \%$, respectively. The application of an auto-catalytic model to the experimental DSC data of each unfilled and filled system, to both neat epoxy resin and graphite-based epoxy nano-composites, leads to kinetic parameters coherent with the envisaged catalyzed reactions.

Dynamic DSC scans also clearly show the the catalytic activity of both eGO and oCB-3, with a fraction of the amine-epoxy catalyzed reaction (based on heating scans at $10 \mathrm{~K} / \mathrm{min}$ ), is close to $29 \%$ and $56 \%$, respectively. Moreover, the main exothermal peak shifts from 113 to 95 and $89{ }^{\circ} \mathrm{C}$, for eGO and oCB-3, respectively.

Hence, the $\mathrm{oCB}$ samples obtained from high specific surface area $\mathrm{CB}$, due to their higher oxidation levels using the same oxidation procedure, exhibit catalytic activity toward the curing of epoxy resins which is definitely higher than for fully exfoliated graphene oxide.

The reported results clearly confirm that oxidized carbon nanomaterials in epoxy-based composites not only act as nanofillers which improve the physical properties, but also as carbocatalysts, suitable for reducing the crosslinking (cure) temperature and/or time of the thermoset matrix.

Acknowledgments: Ministero dell' Istruzione dell' Università e della Ricerca is gratefully acknowledged for financial support.

Author Contributions: Maria Rosaria Acocella and Carola Epsosito Corcione conceived and designed the experiments and participated in the interpretation of the results and the writing of the paper. Antonella Giuri and Mario Maggio were responsible for the experiments and data analysis. Gaetano Guerra and Alfonso Maffezzoli supervised the research and participated in the interpretation of results and the writing of the paper. All the authors contributed to the realization of the manuscript.

Conflicts of Interest: The authors declare no conflict of interest.

\section{References}

1. Jia, H.-P.; Dreyer, D.R.; Bielawski, C.W. C-H oxidation using graphite oxide. Tetrahedron 2011, 67, 4431-4464. [CrossRef]

2. Dreyer, D.R.; Jia, H.-P.; Bielawski, C.W. Graphene oxide: A convenient carbocatalyst for facilitating oxidation and hydration reactions. Angew. Chem. Int. Ed. 2010, 49, 6813-6816. [CrossRef]

3. Boukhvalov, D.V.; Dreyer, D.R.; Bielawski, C.W.; Soon, Y.-W. A computational investigation of the catalytic properties of graphene oxide: Exploring mechanisms by using DFT methods. ChemCatChem 2012, 4, 1844-1849. [CrossRef]

4. Jia, H.-P.; Dreyer, D.R.; Bielawski, C.W. Graphite Oxide as an auto-tandem oxidation-hydration-aldol coupling catalyst. Adv. Synth. Catal. 2011, 353, 528-532. [CrossRef]

5. Kumar, A.V.; Rao, K.R. Recyclable graphite oxide catalyzed Friedel-Crafts addition of indoles to $\alpha, \beta$-unsaturated ketones. Tetrahedron Lett. 2011, 52, 5188-5191. [CrossRef] 
6. Verma, S.; Mungse, H.P.; Kumar, N.; Choudhary, S.; Jain, S.L.; Sain, B.; Khatri, O.P. Graphene oxide: An efficient and reusable carbocatalyst for aza-Michael addition of amines to activated alkenes. Chem. Commun. 2011, 4, 12673-12675. [CrossRef] [PubMed]

7. Acocella, M.R.; Mauro, M.; Falivene, L.; Cavallo, L.; Guerra, G. Inverting the diastereoselectivity of the mukaiyama-michael addition with graphite-based catalysts. ACS Catal. 2014, 4, 492-496. [CrossRef]

8. Acocella, M.R.; De Pascale, M.; Maggio, M.; Guerra, G. Graphite oxide as catalyst for diastereoselective Mukaiyama aldol reaction of 2-(trimethylsilyloxy)furan in solvent free conditions. J. Mol. Catal. A 2015, 408, 237-241. [CrossRef]

9. Dreyer, D.R.; Jarvis, K.A.; Ferriera, P.J.; Bielawski, C.W. Graphite oxide as a dehydrative polymerization catalyst: A one-step synthesis of carbon-reinforced poly(phenylene methylene) composites. Macromolecules 2011, 44, 7659-7667. [CrossRef]

10. Mauro, M.; Acocella, M.R.; Esposito Corcione, C.; Maffezzoli, A.; Guerra, G. Catalytic activity of graphite-based nanofillers on cure reaction of epoxy resins. Polymer 2014, 22, 5612-5615. [CrossRef]

11. Dhakshinamoorthy, A.; Alvaro, M.; Concepcion, P.; Fornes, V.; Garcia, H. Graphene oxide as an acid catalyst for the room temperature ring opening of epoxides. Chem. Commun. 2012, 48, 5443-5445. [CrossRef] [PubMed]

12. Acocella, M.R.; D’Urso, L.; Maggio, M.; Guerra, G. Green regio- and enantioselective aminolysis catalyzed by graphite and graphene oxide under solvent-free conditions. ChemCatChem 2016, 8, 1915-1920. [CrossRef]

13. Roy, B.; Ghosh, S.; Ghosh, P.; Basu, B. Graphene oxide (GO) or reduced graphene oxide (rGO): Efficient catalysts for one-pot metal-free synthesis of quinoxalines from 2-nitroaniline. Tetrahedron Lett. 2015, 56, 6762-6767. [CrossRef]

14. Basu, B.; Kundu, S.; Sengupt, D. Graphene oxide as a carbocatalyst: The first example of a one-pot sequential dehydration-hydrothiolation of secondary aryl alcohols. RSC Adv. 2013, 3, 22130. [CrossRef]

15. Navalon, S.; Dhakshinamoorthy, A.; Alvaro, M.; Garcia, H. Carbocatalysis by graphene-based materials. Chem. Rev. 2014, 14, 6179-6212. [CrossRef] [PubMed]

16. Dhakshinamoorthy, A.; Primo, A.; Concepcion, P.; Alvaro, M.; Garcia, H. Doped graphene as a metal-free carbocatalyst for the selective aerobic oxidation of benzylic hydrocarbons, cyclooctane and styrene. Chem. Eur. J. 2013, 19, 7547-7554. [CrossRef] [PubMed]

17. Primo, A.; Neatu, F.; Florea, M.; Parvulescu, V.; Garcia, H. Graphenes in the absence of metals as carbocatalysts for selective acetylene hydrogenation and alkene hydrogenation. Nat. Commun. 2014, $5,5291$.

18. Acocella, M.R.; Mauro, M.; Guerra, G. Regio- and Enantioselective friedel-crafts reactions of indoles to epoxides catalyzed by graphene oxide: A green approach. ChemSusChem 2014, 7, 3279-3283. [CrossRef] [PubMed]

19. Zhu, S.; Chen, C.; Xue, Y.; Wu, J.; Wang, J.; Fan, W. Graphene oxide: An efficient acid catalyst for alcoholysis and esterification reactions. Chem CatChem 2014, 6, 3080-3083. [CrossRef]

20. Acocella, M.R.; Esposito Corcione, C.; Giuri, A.; Maggio, M.; Maffezzoli, A.; Guerra, G. Graphene oxide as a catalyst for ring opening reactions in amine crosslinking of epoxy resins. RSC Adv. 2016, 6, 23858-23865. [CrossRef]

21. Sangermano, M.; Tagliaferro, A.; Foix, D.; Castellino, M.; Celasco, E. In situ reduction of graphene oxide in an epoxy resin thermally cured with amine. Macromol. Mater. Eng. 2014, 299, 757-763. [CrossRef]

22. Carton, A.; Stevenson, W.T.K.; Wang, S. Interfacial reactions in carbon-epoxy composites. Br. Polym. J. 1987, $19,459$.

23. Nakahara, M.; Takada, T.; Kumagai, H.; Sanada, Y. Surface chemistry of carbon black through curing process of epoxy resin. Carbon 1995, 11, 1537-1544. [CrossRef]

24. Maggio, M.; Acocella, M.R.; Guerra, G. Intercalation compounds of carbon black. RSC Adv. 2016, 6, 105565-105572. [CrossRef]

25. Opalicki, M.; Kenny, J.M.; Nicolais, L. Cure kinetics of neat and carbon-fiber-reinforced TGDDM/DDS epoxy systems. J. Appl. Polym. Sci. 1996, 61, 1025-1037. [CrossRef]

26. Mauro, M.; Cipolletti, V.; Galimberti, M.; Longo, P.; Guerra, G. Chemically reduced graphite oxide with improved shape anisotropy. J. Phys. Chem. C 2012, 116, 24809-24813. [CrossRef]

27. Hummers, W.S.; Offeman, R.E. Preparation of graphitic oxide. J. Am. Chem. Soc. 1958, 80, 1339. [CrossRef] 
28. Esposito, C.; Maffezzoli, A. Glass transition in thermosetting clay-nanocomposite polyurethanes. Thermochim. Acta 2009, 485, 43-48.

29. Laza, J.M.; Julian, C.A.; Larrauri, E.; Rodriguez, M.; Leon, L.M. Thermal scanning rheometer analysis of curing kinetic of an epoxy resin: An amine as curing agent. Polymer 1999, 40, 35-45. [CrossRef]

30. Henning Winter, H.; Chambon, F. Analysis of linear viscoelasticity of a crosslinking polymer at the gel point. J. Rheol. 1986, 30, 367. [CrossRef]

31. Flory, P.J. Principles of Polymer Chemistry; Cornell University Press: Ithaca, NY, USA, 1953.

32. Wei, Z.; Yang, Y.; Hou, Y.; Liu, Y.; He, X.; Deng, S. A new approach towards acid catalysts with high reactivity based on graphene nanosheets. Chem CatChem 2014, 6, 2354-2363. [CrossRef]

33. He, D.; Kou, Z.; Xiong, Y.; Cheng, K.; Chen, X.; Pan, M.; Mu, S. Simultaneous sulfonation and reduction of graphene oxide as highly efficient supports for metal nanocatalysts. Carbon 2014, 66, 312-319. [CrossRef]

34. Maffezzoli, A.; Trivisano, A.; Opalicki, M.; Mijovic, J.; Kenny, J.M. Correlation between dielectric and chemorheological properties during cure of epoxy-based composites. J. Mater. Sci. 1994, 29, 800-808. [CrossRef]

35. Guerrero-Ruiz, A.; Badenes, P.; Rodriguez-Ramos, I. Study of some factors affecting the Ru and Pt dispersions over high surface area graphite-supported catalysts. Appl. Catal. A Gen. 1998, 173, 313-321. [CrossRef]

36. Han, C.D. Developments in Polymer Characterisation-1, 1st ed.; Dawkins, J.V., Ed.; Applied Science: London, UK, 1978; pp. 469-470.

37. Enns, J.B.; Gillhamm, J.K. Time-temperature-transformation (TTT) cure diagram: Modeling the cure behavior of thermosets. J. Appl. Polym. Sci. 1983, 28, 2567-2591. [CrossRef]

38. Maffezzoli, A.; Terzi, R. Thermal analysis of visible-light-activated dental composites. Thermochim. Acta 1995, 269, 319-335. [CrossRef]

39. Ryan, M.E.; Dutta, A. Kinetics of epoxy cure: A rapid technique for kinetic parameter estimation. Polymer 1979, 20, 203-206. [CrossRef]

40. Kamal, M.R.; Sourour, S. Kinetics and thermal characterization of thermoset cure. Polym. Eng. Sci. 1973, 13, 59-64. [CrossRef]

41. Cole, K.C. A new approach to modeling the cure kinetics of epoxy/amine thermosetting resins: Mathematical development. Macromolecules 1991, 24, 3093-3097. [CrossRef]

42. Cole, K.C.; Hechler, J.J.; Noel, D. A new approach to modeling the cure kinetics of epoxy/amine thermosetting resins. 2. Application to a typical system based on bis[4-(diglycidylamino)phenyl]methane and bis(4-aminophenyl) sulfone. Macromolecules 1991, 24, 3098-3110. [CrossRef]

43. Kenny, J.M.; Apicella, A.; Nicolais, L. A model for the thermal and chemorheological behavior of thermosets. I: Processing of epoxy-based composites. Polym. Eng. Sci. 1989, 29, 973-983. [CrossRef]

44. Trivisano, A.; Maffezzoli, A.; Kenny, J.M.; Nicolais, L. Mathematical modeling of the pultrusion of epoxy based composites. Adv. Polym. Technol. 1990, 4, 251-264. [CrossRef]

45. Xie, H.; Liu, B.; Yuan, Z.; Shen, J.; Cheng, R. Cure kinetics of carbon nanotube/tetrafunctional epoxy nanocomposites by isothermal differential scanning calorimetry. J. Polym. Sci. Part B 2004, 42, 3701-3712. [CrossRef]

(C) 2017 by the authors. Licensee MDPI, Basel, Switzerland. This article is an open access article distributed under the terms and conditions of the Creative Commons Attribution (CC BY) license (http://creativecommons.org/licenses/by/4.0/). 